\title{
Microcavity effects in the photoluminescence of hydrogenated amorphous silicon nitride
}

Ali Serpenguzel

Atilla Aydinli

Alpan Bek 


\title{
Microcavity effects in the photoluminescence of hydrogenated amorphous silicon nitride
}

\author{
Ali Serpengüzel, ${ }^{a}$ Atilla Aydinli, ${ }^{\text {a,b }}$ and Alpan Bek ${ }^{\text {a,b }}$ \\ ${ }^{a}$ Permanent adress: Bilkent University, Physics Department, Bilkent, Ankara 06533 Turkey \\ ${ }^{b}$ University of California, Electrical and Computer Engineering Department, \\ Santa Barbara, California 93 106-9560 USA
}

\begin{abstract}
Fabry-Perot microcavities are used for the alteration of photoluminescence in hydrogenated amorphous silicon nitride grown with and without ammonia. The photoluminescence is red-near-infrared for the samples grown without ammonia, and blue-green for the samples grown with ammonia. In the Fabry-Perot microcavities, the amplitude of the photoluminescence is enhanced, while its linewidth is reduced with respect to the bulk hydrogenated amorphous silicon nitride. The microcavity was realized by a metallic back mirror and a hydrogenated amorphous silicon nitride - air or a metallic front mirror. The transmittance, reflectance, and absorbance spectra were also measured and calculated. The calculated spectra agree well with the experimental spectra. The hydrogenated amorphous silicon nitride microcavity has potential for becoming a versatile silicon based optoelectronic device such as a colot flat panel display, a resonant cavity enhanced light emitting diode, or a laser.
\end{abstract}

Keywords: microcavity, Fabry-Perot, amorphous silicon, photoluminescence, resonators, thin films, spontaneous emission, optoelectronics, plasma enhanced chemical vapor deposition.

\section{INTRODUCTION}

Interest in silicon ( $\mathrm{Si}$ ) as a material for optoelectronics has increased recently. With modern process techniques, it will be possible to integrate lasers, photodetectors, and waveguides on optoelectronic silicon motherboards. Hydrogenated amorphous silicon (a-Si:H) ${ }^{\prime}$ has been used for the realization of planar waveguides, which will be able to route and modulate optical signals within such a silicon motherboard. An advantage of a-Si:H is that, it can be deposited by plasma enhanced chemical vapor deposition (PECVD) on almost any substrate at temperatures below $500 \mathrm{~K}$, which makes it compatible with the microelectronic technology. Another advantage of the amorphous silicon ${ }^{2}$ as well as porous silicon ${ }^{3}$ is that, they attract interest as a potential optical gain medium because of their room temperature visible electroluminescence and photoluminescence $(\mathrm{PL})$.

Microcavities also continue to attract attention, due to their unique optical properties. ${ }^{4}$ In a microcavity, two electromagnetic and quantum electrodynamic effects occur. First, the microcavity acts as an optical resonator for light rays with specific wavelengths, which after one round trip, return to their starting position in phase, i.e., resonate in the microcavity. Second, in a microcavity, the photon density of states are enhanced at the cavity resonances, when compared with the continuum of photon states of a bulk sample. The spontaneous emission (luminescence) cross-sections (quantum efficiencies) at the microcavity resonances are larger than the bulk spontaneous emission cross-sections because of the enhanced photon density of states. ${ }^{5}$ Also, the luminescence cross-sections in between the microcavity resonances are

Further author information -

A. S. (correspondence) : E-mail: serpen(a)fen.bilkent.edu.tr; WWW: http//www.fen.bilkent.edu.tr/ serpen; Telephone: 90 (312) 2664000 Ext. 1965; Fax: 90 (312) 266-4579

A. A. E-mail: aydinli(a)fen.bilkent.edu.tr; WWW: http//www.fen.bilkent.edu.tr/ aydinli; Telephone: 90 (312) 266-4000 Ext. 1579; Fax: 90 (312) 266-4579

A. B. E-mail: bek(@)fen.bilkent.edu.tr; WWW: http//www.fen.bilkent.edu.tr/ bek; Telephone: 90 (312) 266-4000 Ext. 1972 ; Fax: 90 (312) 266-4579 
smaller than the bulk spontaneous emission cross-sections. Alteration (i.e., enhancement and inhibition) of spontaneous emission in planar microcavities has been both observed experimentally ${ }^{6}$ and calculated theoretically. ${ }^{7}$ These properties of the microcavities are used in resonant cavity enhanced (RCE) photonic devices, which are wavelength selective and ideal for wavelength division multiplexing (WDM) applications, ${ }^{8}$ or color flat panel displays. In vertical cavity surface emitting lasers (VCSEL's), ${ }^{9}$ as well as microdisk, ${ }^{10}$ and microwire ${ }^{11}$ lasers, the lasing threshold is reduced and the mode linewidth is narrowed. Similarly, in RCE light emitting diodes (LED's) the efficiency, brightness, and directivity are enhanced. ${ }^{12}$ Also, in RCE photodiodes, ${ }^{8}$ the quantum efficiency is enlanced.

Planar microcavity effects on the PL of porous silicon ${ }^{13}$ as well as $\mathrm{Si} / \mathrm{SiOx}$ superlattices ${ }^{14}$ have already been reported. Recently, we have observed visible PL from a-Si:H, as well as its oxides (a-SiOx:H) and nitrides $(a-S i N x: H)$ grown by low temperature PECVD. ${ }^{15.16}$ In this paper, we report, the enhancement and inhibition of PL in an a-SiNx:H Fabry-Perot microcavity. The a-SiNx:H used in the microcavity is grown both with and without ammonia $\left(\mathrm{NH}_{3}\right)$. For the samples grown without $\mathrm{NH}_{3}$ the $\mathrm{PL}$ is in the red-near-infrared part of the optical spectrum, while for the samples grown with $\mathrm{NH}_{3}$ the PL is in the blue-green part of the optical spectrum. ${ }^{17}$ While the exact mechanism of the occurrence of the PL in bulk a-SiNx:H is still under discussion, we have suggested in Ref. 17 the use of a quantum confinement model. ${ }^{18}$ There, it was proposed that, our samples consist of small a-Si clusters in a matrix of a-SiNx:H. The regions with $\mathrm{Si}-\mathrm{H}$ and $\mathrm{Si}-\mathrm{N}$, having larger energy gaps due to strong $\mathrm{Si}-\mathrm{H}$ and $\mathrm{Si}-\mathrm{N}$ bonds, isolate these a-Si clusters, and form barrier regions around them. The PL originates from the a-Si clusters.

\section{EXPERIMENTAL ARRANGEMENT}

\subsection{Hydrogenated amorphous silicon nitride (a-SiNx:H) growth without $\mathrm{NH}_{3}$}

In the samples grown without $\mathrm{NH}_{3}$, the microcavity was realized by a Au back mirror and an a-SiNx:H-air interface front mirror. First, the thin glass substrates were coated with $100 \mathrm{~nm}$ of Au. Second, a thin layer of a-SiNx:H was deposited on the Au coated substrates by PECVD. The flow rate of the PECVD gas $\left[2 \%\right.$ silane $(\mathrm{SiH})$ in nitrogen $\left.\left(\mathrm{N}_{2}\right)\right]$ was $180 \mathrm{sccm}$, the radio frequency power $10 \mathrm{~W}$, and the deposition chamber pressure 1 Torr. The metric thickness (L) of the a$\mathrm{SiNx}: \mathrm{H}$ layer grown without $\mathrm{NH}_{3}$ was measured to be $1400 \pm 100 \mathrm{~nm}$.

\subsection{Hydrogenated amorphous silicon nitride (a-SiNx:H) growth with $\mathrm{NH}_{3}$}

In the samples grown with $\mathrm{NH}_{3}$, the microcavity was realized by an Al back mirror and an a-SiNx:H-air interface front mirror or a a-SiNx:H-Al partially transmitting front mirror. First, the thin Si or quartz substrates were coated with $100 \mathrm{~nm}$ of $\mathrm{Al}$. Second, a thin layer of a-SiNx:H was deposited on the Al coated substrates by PECVD. The flow rates of the PECVD gases were $180 \mathrm{sccm}$ for $2 \% \mathrm{SiH}_{4}$ in $\mathrm{N}_{2}$ and $10 \mathrm{sccm}$ for $\mathrm{NH}_{3}$, the radio frequency power $10 \mathrm{~W}$, and the deposition chamber pressure 1 Torr. The metric thickness (L) of the a-SiNx:H layer grown with $\mathrm{NH}_{3}$ was measured to be $1000 \pm 100$ $\mathrm{nm}$. Finally, the samples were annealed in a forming gas atmosphere at $600^{\circ} \mathrm{C}$ for $10 \mathrm{~min}$. For the $\mathrm{Al}$ partially transmitting front metallic mirror microcavities, a thin $(\sim 10 \mathrm{~nm})$ layer of $\mathrm{Al}$ was deposited on the annealed samples.

\subsection{Room temperature reflectance, transmittance, and photoluminescence measurements of a-SiNx:H}

The room temperature reflectance and transmittance measurements were made at $0^{\circ}$ with respect to the surface normal with a resolution of $2 \mathrm{~nm}$. The room temperature PL spectra were measured with a resolution of $0.1 \mathrm{~nm}$. The PL spectra were later corrected for the responsivity of the spectrometer and the photomultiplier tube. An $\mathrm{Ar}^{+}$laser with a wavelength of $514.5 \mathrm{~nm}(457.9 \mathrm{~nm})$ and a power of $420 \mathrm{~mW}$ was focused with a $15 \mathrm{~cm}$ focal length cylindrical lens on the samples grown without (with) $\mathrm{NH}_{3}$. The $\mathrm{PL}$ spectra were taken at $0 \pm 3.6^{\circ}$ with the laser at $30^{\circ}$ with respect to the surface normal. During the PL measurements the temperature of the sample is not controlled and there might be local heating due to the poor thermal conductivity of the glass substrate. Local heating reduces the PL efficiency and broadens the PL linewidth. ${ }^{19}$ However, the local heating would not considerably affect the general shape and features of the gain spectrum. As will be seen in the PL spectra, even though there might be local heating, we are observing strong PL from the samples and the PL is enhanced by the Fabry-Perot resonances. 


\section{EXPERIMENTAL RESULTS AND DISCUSSION}

\subsection{Hydrogenated amorphous silicon nitride (a-SiNx:H) grown without $\mathrm{NH}_{3}$}

\subsubsection{Transmittance, reflectance, and absorbance spectra of the a-SiNx:H grown without $\mathrm{NH}_{3}$}

Figures 1, 2, and 3 show the measured and calculated transmittance, reflectance, and absorbance spectra of the aSiNx:H grown without $\mathrm{NH}_{3}$, respectively. The absorbance spectrum is obtained by subtracting both the reflectance and transmittance spectra from unity, i.e., $100 \%$. In the calculations, the total transmitted and the reflected electric fields for each wavelength were obtained using an absorbing Fabry-Perot microcavity model. In this model, we assumed the sample to be a one dimensional absorbing dielectric slab on a glass substrate. The experimentally measured absorbance spectrum was obtained by subtracting both the experimentally measured transmittance and the reflectance spectra from $100 \%$. This experimentally measured absorbance spectrum was then incorporated to the calculations of the transmitted and reflected electric fields. The calculated transmittance and reflectance at each wavelength were obtained from their respective electric fields. The calculated transmittance and reflectance intensity was subtracted from $100 \%$ to obtain the calculated absorbance intensity as a final check.

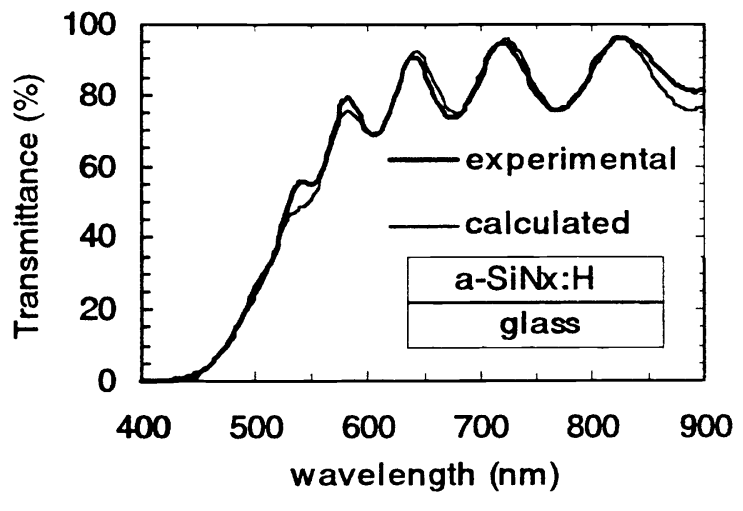

Fig. 1. Experimental and theoretical transmittance spectra of the a-SiNx:H grown without $\mathrm{NH}_{3}$.

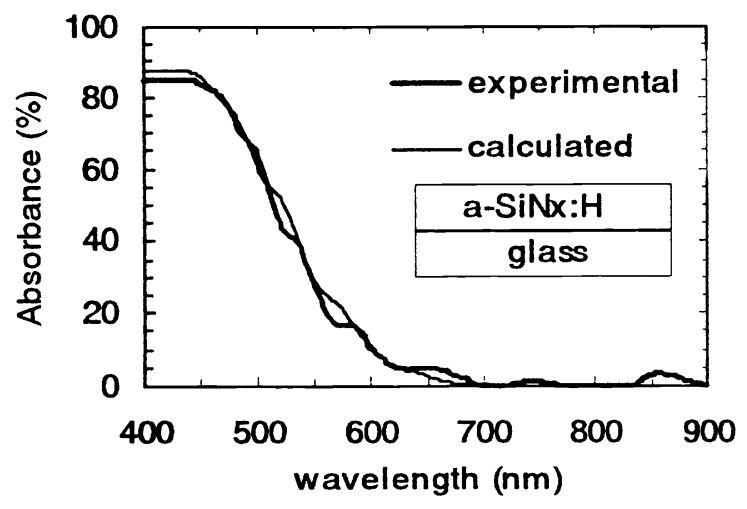

Fig. 3. Experimental and calculated absorbance spectrum of a-SiNx:H grown without $\mathrm{NH}_{3}$.

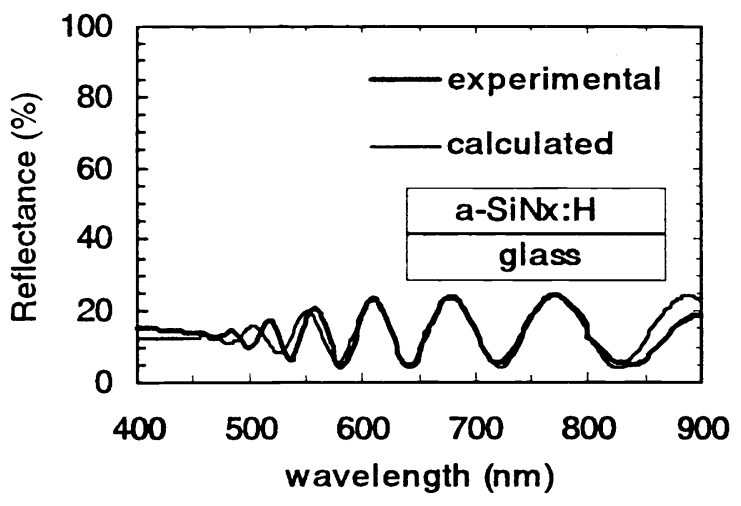

Fig. 2. Experimental and theoretical reflectance spectra of the a-SiNx:H grown without $\mathrm{NH}_{3}$.

The theoretical fit to the experimental spectra is extremely good. The metric thickness of the a-SiNx:H is found to be $L=1376 \mathrm{~nm}$ (which agrees well with the experimental thickness of $1400 \pm 100 \mathrm{~nm}$ ) and the refractive index $n=2.1$. Both the transmittance and the reflectance spectra show Fabry-Perot resonances at wavelengths satisfying the condition $\left(\lambda_{\mathrm{m}}=2 \mathrm{Ln} / \mathrm{m}\right.$, where $\mathrm{m}$ is the quantized mode number). The mode number of these resonances were found to range from $m=11\left(\lambda_{11}=545 \mathrm{~nm}\right)$ to $\mathrm{m}=7\left(\lambda_{7}=835 \mathrm{~nm}\right)$. The resonances have linewidths of $\Delta \lambda=35 \mathrm{~nm}$ and quality factors of $\mathrm{Q}=20$. Below $600 \mathrm{~nm}$, the resonances start to wash out by the absorption of the aSiNx:H. The loading of the resonances by the a-SiNx:H absorption stops above $600 \mathrm{~nm}$. 


\subsubsection{Photoluminescence spectra of the a-SiNx: $\mathrm{H}$ grown without $\mathrm{NH}_{3}$}

Figure 4 shows the $\mathrm{PL}$ of the a-SiNx:H grown without $\mathrm{NH}_{3}$, whose transmittance, reflectance, and absorbance spectra were shown in Figs. 1, 2, and 3. The PL intensity is modulated by the weak Fabry-Perot resonances, which correlate well with the maxima of the transmittance spectrum of Fig. 1, and the minima of the reflectance spectrum of Fig. 2. The rednear-infrared PL has a broad linewidth $(240 \mathrm{~nm})$. This broad linewidth of the a-SiNx:H shows that, a-SiNx:H has potential as a novel photonic gain medium. Figure 5 depicts the PL of the a-SiNx:H microcavity with the Au back mirror. The Fabry-Perot resonances have experimental and theoretical linewidths of $\Delta \lambda=25 \mathrm{~nm}$ and quality factors of $\mathrm{Q}=30$. The metric thickness of the a-SiNx:H microcavity with the Au mirror was found to be $\mathrm{L}=1438 \mathrm{~nm}$ (which agrees well with the experimental thickness of $1400 \pm 100 \mathrm{~nm}$ ) and the refractive index $n=2.1$. The PL is modulated by the strong Fabry-Perot resonances. The mode number of these resonances were found to range from $\mathrm{m}=11\left(\lambda_{11}=558 \mathrm{~nm}\right)$ to $\mathrm{m}=7\left(\lambda_{7}=874\right.$ $\mathrm{nm})$.

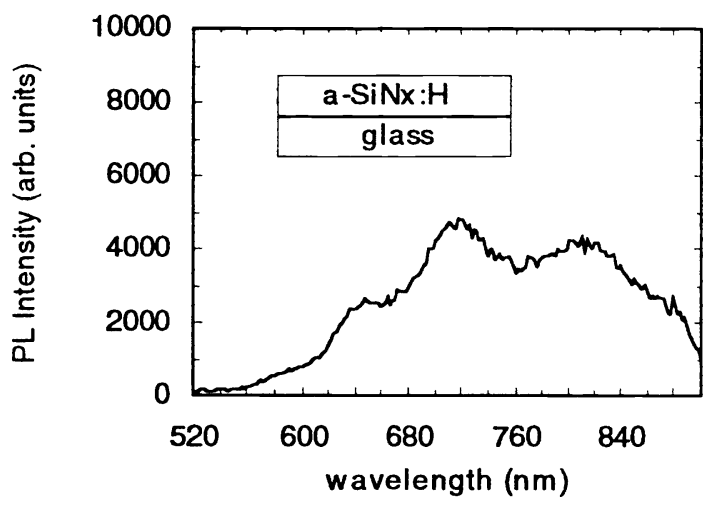

Fig.4. PL spectrum of the a-SiNx:H grown without $\mathrm{NH}_{3}$.

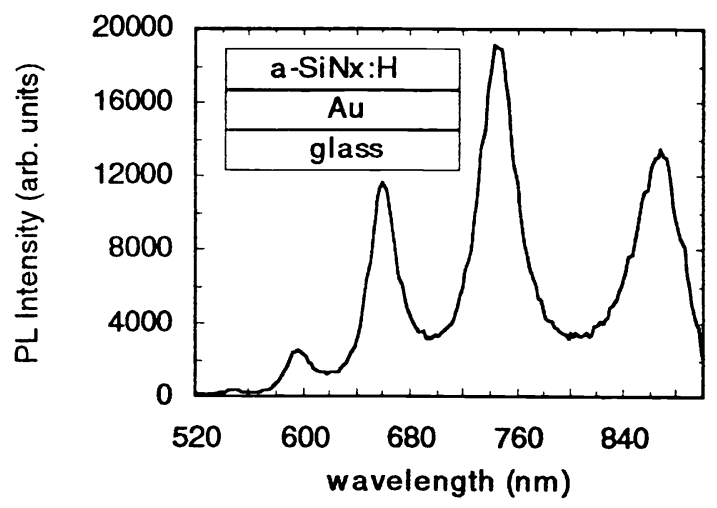

Fig.5. PL of the a-SiNx:H grown with $\mathrm{NH}_{3}$ with the Au mirror .

The two PL spectra of Figs. 4 and 5 were obtained under the same experimental conditions. When comparing the spectra in Figs. 4 and 5, the PL of the microcavity with the Au mirror has 3 noteworthy features with respect to the PL of the a-SiNx:H : (1) there is a $2 \mathrm{X}$ increase of the overall spectrum average (i.e., averaging out the Fabry-Perot resonances), (2) there is a $4 \mathrm{X}$ enhancement of the PL peaks, and (3) the PL dips have similar amplitude. These 3 features are noticed with respect to the PL of the a-SiNx:H. The $2 \mathrm{X}$ increase is due to the "round trip" of the excitation $\mathrm{Ar}^{+}$laser in the Fabry Perot cavity due to the back Au mirror. Since the wavelength of the $\mathrm{Ar}^{+}$laser is non-resonant, the input laser light does not resonate in the cavity, which would have enhanced the PL further. The $4 \mathrm{X}$ enhancement at the resonances, are clearly due to the combined effect of the enhancement of the PL by the cavity resonances with that of the input laser reflecting from the back Au mirror. The PL dips having the same amplitude in both spectra is due to the inhibition of the PL in between the resonances.

\subsection{Hydrogenated amorphous silicon nitride (a-SiNx:H) grown with $\mathrm{NH}_{3}$}

\subsubsection{Transmittance, reflectance, and absorbance spectra of the a-SiNx: $\mathrm{H}$ grown with $\mathrm{NH}_{3}$}

Figures 6, 7, and 8 show the measured and calculated transmittance, reflectance, and absorbance spectra of the aSiNx:H grown with $\mathrm{NH}_{3}$, respectively. The absorbance spectrum is obtained by subtracting both the reflectance and transmittance spectra from unity, i.e., $100 \%$. In the calculations, the total transmitted and the reflected electric fields for each wavelength were obtained using an absorbing Fabry-Perot microcavity model. In this model, we assumed the sample to be a one dimensional absorbing dielectric slab on a quartz substrate. The experimentally measured absorbance spectrum was obtained by subtracting both the experimentally measured transmittance and the reflectance spectra from $100 \%$. This experimentally measured absorbance spectrum was then incorporated to the calculations of the transmitted and reflected electric fields. The calculated transmittance and reflectance at each wavelength were obtained from their respective electric fields. The calculated transmittance and reflectance intensity was subtracted from $100 \%$ to obtain the calculated absorbance intensity as a final check. 


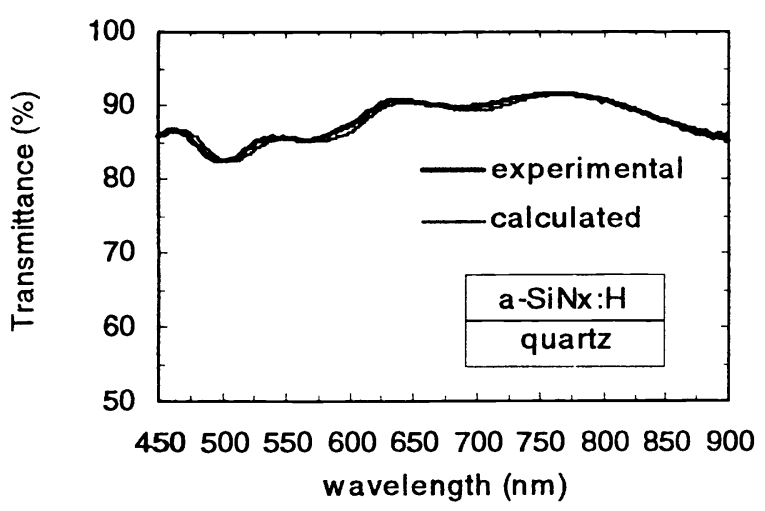

Fig. 6. Experimental and theoretical transmittance spectra of the a-SiNx:H grown with $\mathrm{NH}_{3}$.

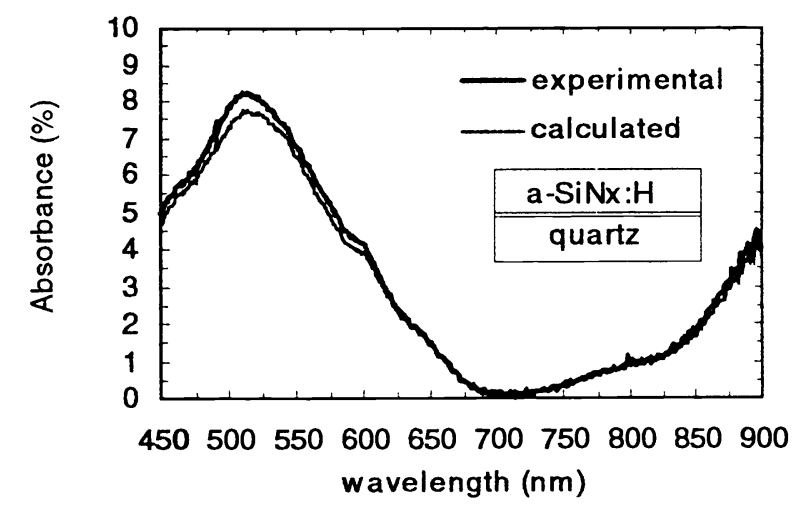

Fig. 8. Experimental and calculated absorbance spectrum of a-SiNx:H grown with $\mathrm{NH}_{3}$.

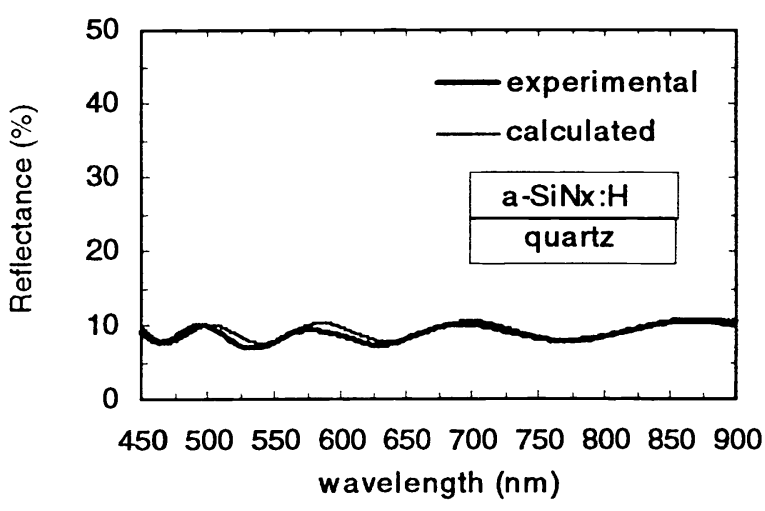

Fig. 7. Experimentally measured and theoretically calculated reflectance spectra of the a-SiNx:H grown with $\mathrm{NH}_{3}$.

The theoretical fit to the experimental spectra is extremely good. The metric thickness of the a-SiNx:H grown with $\mathrm{NH}_{3}$ is found to be $\mathrm{L}=963 \mathrm{~nm}$ (which agrees well with the experimental thickness of $1000 \pm 100 \mathrm{~nm}$ ) and the refractive index $n=1.6$. Both the transmittance and the reflectance spectra show Fabry-Perot resonances at wavelengths satisfying the condition $\left(\lambda_{m}=2 \mathrm{Ln} / \mathrm{m}\right.$, where $\mathrm{m}$ is the quantized mode number). The mode number of these resonances were found to range from $\mathrm{m}=7\left(\lambda_{7}=461 \mathrm{~nm}\right)$ to $\mathrm{m}=4\left(\lambda_{4}=760 \mathrm{~nm}\right)$. The resonances have linewidths of $\Delta \lambda=40 \mathrm{~nm}$ and quality factors of $\mathrm{Q}=10$. Compared with the absorption of the a-SiNx:H grown without $\mathrm{NH}_{3}$, the absorption of the a-SiNx:H grown with $\mathrm{NH}_{3}$ is an order of magnitude lower. Consequently, there is no loading of the resonances by the absorption.

\subsubsection{Photoluminescence spectra of the a-SiNx:H grown with $\mathrm{NH}_{3}$}

Figure 9 shows the $\mathrm{PL}$ of the a-SiNx:H grown with $\mathrm{NH}_{3}$ on $\mathrm{Si}$ together with the samples grown on quartz, whose transmittance, reflectance, and absorbance spectra were shown in Figs. 6, 7, and 8. The samples grown on quartz are used for transmittance, and reflectance measurements. The samples grown on Si are used for PL measurements, since Al sticks better to Si when compared with quartz. The PL intensity in Fig. 9 is modulated by the weak Fabry-Perot resonances. The mode number of these resonances were found to range from $\mathrm{m}=7\left(\lambda_{7}=488 \mathrm{~nm}\right)$ to $\mathrm{m}=6\left(\lambda_{6}=572 \mathrm{~nm}\right)$. The PL is inhibited in between these two resonances at $\mathrm{m}=6.5\left(\lambda_{6.5}=524 \mathrm{~nm}\right)$. The blue-green PL has also a broad linewidth (100 $\mathrm{nm}$ ). This broad linewidth of the a-SiNx:H grown with $\mathrm{NH}_{3}$ again shows that, a-SiNx:H has potential as a novel photonic gain medium. The Fabry-Perot resonances have experimental and theoretical linewidths of $\Delta \lambda=40 \mathrm{~nm}$ and quality factors of $Q=10$. The metric thickness of the a-SiNx:H grown on Si was found to be $L=1103 \mathrm{~nm}$ (which agrees well with the experimental thickness of $1000 \pm 100 \mathrm{~nm}$ ) and the refractive index $n=1.6$.

Figure 10 depicts the PL of the a-SiNx:H microcavity with the $\mathrm{Al}$ back mirror. Al is chosen instead of Au because of its high reflectance in the blue-green part of the optical spectrum. The Fabry-Perot resonances have experimental and theoretical linewidths of $\Delta \lambda=60 \mathrm{~nm}$ and quality factors of $\mathrm{Q}=8$. The metric thickness of the a-SiNx:H microcavity with the $\mathrm{Al}$ mirror was found to be $\mathrm{L}=929 \mathrm{~nm}$ (which agrees well with the experimental thickness of $1000 \pm 100 \mathrm{~nm}$ ) and the refractive index $\mathrm{n}=1.6$. The PL is modulated by the strong Fabry-Perot resonances. The mode number of these resonances were found to range from $\mathrm{m}=6\left(\lambda_{6}=515 \mathrm{~nm}\right)$ to $\mathrm{m}=5\left(\lambda_{5}=603 \mathrm{~nm}\right)$. 


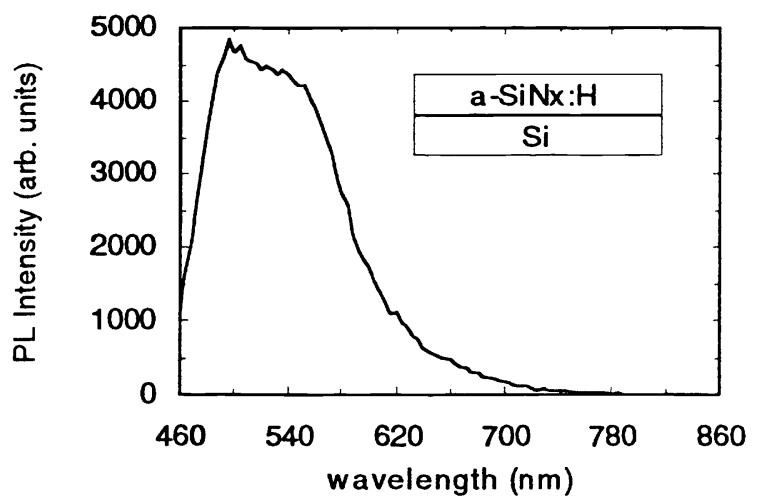

Fig. 9. PL spectrum of the a-SiNx:H grown with $\mathrm{NH}_{3}$.

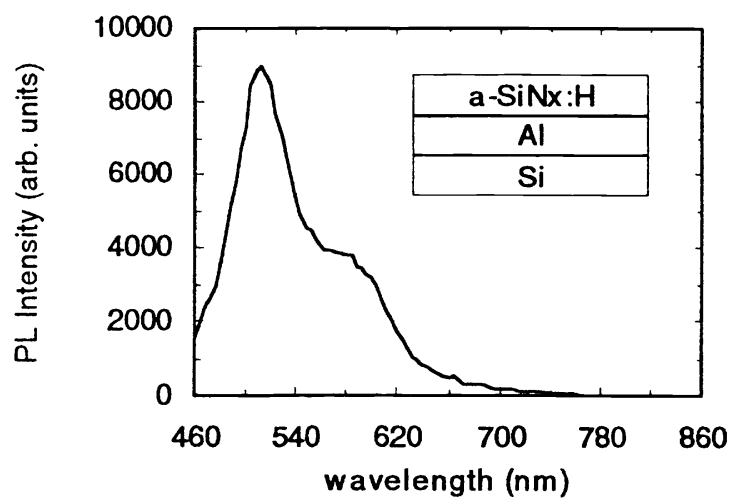

Fig. 10. PL spectrum of the a-SiNx:H grown with $\mathrm{NH}_{3}$ with the Al back mirror.

The two PL spectra of Figs. 9 and 10 were obtained under the same experimental conditions. When comparing the spectra in Figs. 9 and 10, the PL of the microcavity with the Al mirror has 3 noteworthy features with respect to the PL of the a-SiNx:H: (1) there is a $1.3 \mathrm{X}$ increase of the overall spectrum average (i.e., averaging out the Fabry-Perot resonances), (2) there is a $2 X$ enhancement of the PL peaks, and (3) the PL dips have similar amplitude. These 3 features are noticed with respect to the PL of the a-SiNx:H. The $1.3 \mathrm{X}$ increase is due to the "round trip" of the excitation $\mathrm{Ar}^{+}$laser in the Fabry Perot cavity due to the presence of the back $\mathrm{Al}$ mirror instead of a Si substrate, which only partially reflects the incident beam. Since the wavelength of the $\mathrm{Ar}^{+}$laser is non-resonant, the input laser light does not resonate in the cavity, which would have enhanced the PL further. The $2 \mathrm{X}$ enhancement at the resonances, are clearly due to the combined effect of the enhancement of the PL by the cavity resonances with that of the input laser reflecting from the back Al mirror. The PL dips having the same amplitude in both spectra is due to the inhibition of the PL in between the resonances.

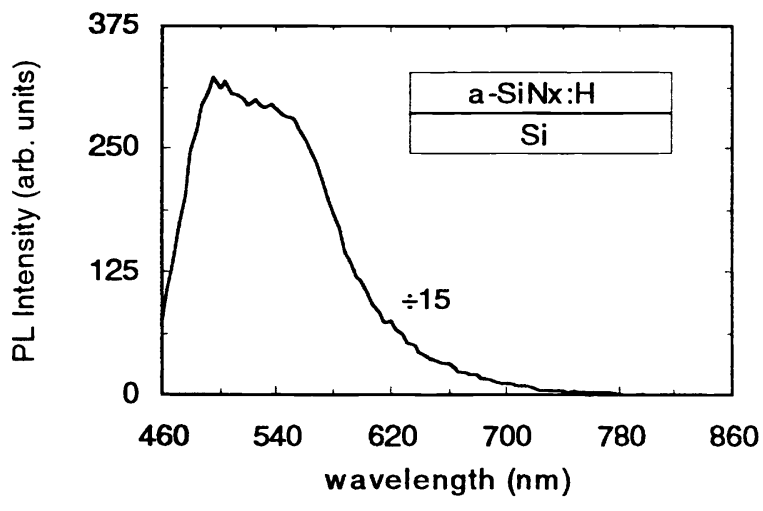

Fig. 11. PL spectrum of the a-SiNx:H grown with $\mathrm{NH}_{3}$ divided by 15 .

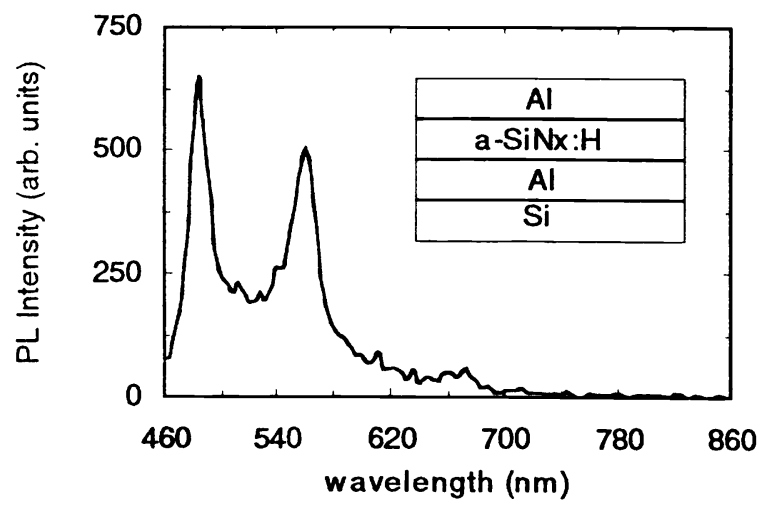

Fig. 12. P'L spectrum of the a-SiNx:H grown with $\mathrm{NH}_{3}$ with the Al back and front mirrors.

Figure 11 shows the $\mathrm{PL}$ of the a-SiNx:H grown with $\mathrm{NH}_{3}$ (same as Fig. 9) divided by 15 , in order to have the same spectral average for both Figs. 11 and 12. Figure 12 depicts the PL of the a-SiNx:H microcavity with the $\mathrm{Al}$ back and $\mathrm{Al}$ front partial mirror. The Fabry-Perot resonances have experimental and theoretical linewidths of $\Delta \lambda=20 \mathrm{~nm}$ and quality factors of $\mathrm{Q}=25$. The metric thickness of the a-SiNx:H microcavity with the $\mathrm{Al}$ back and $\mathrm{Al}$ front partial mirror was found to be $\mathrm{L}=1072 \mathrm{~nm}$ (which agrees well with the experimental thickness of $1000 \pm 100 \mathrm{~nm}$ ) and the refractive index $\mathrm{n}$ $=1.6$. The PL is modulated by the strong Fabry-Perot resonances. The mode number of these resonances were found to range from $\mathrm{m}=7\left(\lambda_{7}=488 \mathrm{~nm}\right)$ to $\mathrm{m}=5\left(\lambda_{5}=687 \mathrm{~nm}\right)$. 
The PL spectra of Fig. 12 was obtained under the same experimental conditions as of Fig. 11. When comparing the spectra in Figs. 11 and 12, the PL of the microcavity with the Al back and front partial mirror has 3 noteworthy features with respect to the PL of the a-SiNx:H: (1) the overall spectral averages are the same (i.e., averaging out the Fabry-Perot resonances), (2) there is a $2 X$ enhancement of the PL peaks, and (3) the PL dips have similar amplitude. These 3 features are noticed with respect to the PL of the a-SiNx:H. The $2 \mathrm{X}$ enhancement at the resonances, while the overall average being the same, are clearly due to the enhancement of the PL by the cavity resonances. The PL dips having the same amplitude in both spectra is due to the inhibition of the PL in between the resonances.

\section{CONCLUSIONS}

In conclusion, Fabry-Perot microcavities with dielectric and metallic mirrors are used for the alteration of photoluminescence in a-SiNx:H grown with and without $\mathrm{NH}_{3}$. The photoluminescence of the bulk a-SiNx:H is in the rednear-infrared part of the optical spectrum for the samples grown without $\mathrm{NH}_{3}$. However, the photoluminescence of the bulk a-SiNx:H is in the blue-green part of the optical spectrum for the samples grown with $\mathrm{NH}_{3}$. These complementary colors cover the whole visible spectrum, and one can imagine a color flat panel display made out of a-SiNx:H pixels. The broad photoluminescence spectrum of the a-SiNx:H, also makes it a suitable source for WDM applications.

The photoluminescence of the bulk a-SiNx:H is enhanced at, and inhibited in between, the Fabry-Perot resonances of the microcavity. The enhancement and inhibition of the photoluminescence is understood by the modified photon density of states of the microcavity. The linewidth of the photoluminescence is also narrowed with respect to the linewidth of the bulk a-SiNx:H, again due to the presence of the electromagnetic modes of the microcavity. The Fabry-Perot enhancement and inhibition of luminescence in a-SiNx:H opens up a variety of possibilities for optoelectronic applications such as color flat panel displays, resonant cavity enhanced (RCE) light emitting diodes (LED's), or microlasers.

\section{ACKNOWLEDGMENTS}

We would like to thank Murat Güre of Bilkent University, Physics Department for his help with the growth of the samples. We would also like to thank Prof. Richard K. Chang of Yale University, Applied Physics Department for many helpful discussions and reading of the manuscript. We would like to acknowledge the partial support of this research by the Scientific and Technical Research Council of Turkey (TÜBITAK) Grant No: TBAG-1368 and the International Center for Theoretical Physics (ICTP) Grant No: 95-500 RG/PHYS/AS.

\section{REFERENCES}

1. A. M. Agarwal, L. Liao, J. S. Foresi, M. R. Black, X. Duan, and L.C. Kimerling, "Low-loss Polycrystalline Silicon Waveguides for Silicon Photonics," J. Appl. Phys. 80, 6120 (1996).

2. D. J. Lockwood, "Optical Properties of Porous Silicon," Solid State (Commun. 92, 101 (1994).

3. T. Canham, "Silicon Quantum Wire Array Fabrication by Electrochemical and Chemical Dissolution of Wafers," Appl. Phys. Lett. 57, 1046 (1990).

4. R. K. Chang and A. J. Campillo, Eds., Optical Processes in Microcavities (World Scientific, Singapore, 1996).

5. E. M. Purcell, " Spontaneous Emission Probabilities at Radio Frequencies, "Phys. Rev. 69, 681 (1946).

6. F. De Martini, G. Innocenti, G. R. Jacobowitz, and P. Mataloni, "Anomalous Spontaneous Emission Time in a Microscopic Optical Cavity," Phys. Rev. Lett. 59, 2955 (1987).

7. H. Yokoyama, S. D. Brorson, "Rate Equation Analysis of Microcavity Lasers," J. Appl. Phys. 66, 4801 (1989).

8. M. S. Ünlü and S. Strite, "Resonant Cavity Enhanced Photonic Devices," J. Appl. Phys. 78, 607 (1995).

9. H. Yokoyama, K. Nishi, T. Anan, H. Yamada, S. D. Brorson, and E. P. Ippen, "Enhanced Spontaneous Emission from GaAs quantum Wells in Monolithic Microcavities," Appl. Phys. Lett. 57, 2814 (1990).

10.S. L. McCall, A. F. J. Levi, R. E. Slusher, S. J. Pearton, and R. A. Logan, "Whispering Gallery Mode Microdisk Lasers," Appl. Phys. Lett. 60, 289 (1992).

11.J. P. Zhang, D. Y. Chu, S. L. Wu, S. T. Ho, W. G. Bi, C. W. Tu, and R. C. Tiberio, "Photonic Wire Laser," Phys. Rev. Lett. 75, 2678 (1995).

12. E. F. Schubert, Y.-H. Wang, A. Y. Cho, 1. W. Tu, and G. J. Zydzik, "Resonant Cavity Light Emitting Diode," Appl. Phys. Lett. 60, 921 (1992). 
13.L. Pavesi, R. Guardini, and C. Mazolleni, "Porous Silicon Resonant Cavity Light Emitting Diodes," Solid State Commun. 97, 1051 (1996).

14.B. T. Sullivan, D. J. Lockwood, H. J. Labbe, and Z.-H. Lu, "Photoluminescence in Amorphous Si/SiOx Superlattices Fabricated by Magnetron Sputtering," Appl. Phys. Lett. 693149 (1996).

15.F. N. Timofeev, A. Aydinli, R. Ellialtioglu, K. Türkoglu, M. Güre, V. N. Mikhailov, and O. A. Lavrova, "Visible Photoluminescence from SiOx Films Grown by Low Temperature Plasma Enhanced Chemical Vapor Deposition," Solid State Commun. 95, 443 (1995).

16. A. Serpengiizel, A. Aydinli, and A. Bek, "Enhancement and inhibition of photoluminescence in hydrogenated amorphous Silicon nitride microcavities, " Optics Express 1, 108 (1997).

17. A. Aydinli, A. Serpengüzel, and D. Vardar, "Visible Photoluminescence from Low Temperature Deposited Hydrogenated Amorphous Silicon Nitride," Solid State Commun. 98, 273 (1996).

18. M. H. Brodsky, "Quantum Well Model of the Hydrogenated Amorphous Silicon," Solid State Commun. 36, 55 (1980).

19. R. Fisher, in Amorphous Semiconductors, M. H. Brodsky, ed. (Springer-Verlag, Berlin, 1985). pp.159-187. 